\title{
Evaluating the performance of sentence level features and domain sensitive features of product reviews on supervised sentiment analysis tasks
}

\author{
Bagus Setya Rintyarna ${ }^{1,2^{*}} \mathbb{D}$, Riyanarto Sarno ${ }^{1}$ and Chastine Fatichah ${ }^{1}$
}

\section{${ }^{*}$ Correspondence:}

bagus.setya@unmuhjember. ac.id

${ }^{2}$ Department of Electrical Engineering, Universitas Muhammadiyah Jember, Jember, Indonesia Full list of author information is available at the end of the article

\begin{abstract}
With the popularity of e-commerce, posting online product reviews expressing customer's sentiment or opinion towards products has grown exponentially. Sentiment analysis is a computational method that plays an essential role in automating the extraction of subjective information i.e. customer's sentiment or opinion from online product reviews. Two approaches commonly used in Sentiment analysis tasks are supervised approaches and lexicon-based approaches. In supervised approaches, Sentiment analysis is seen as a text classification task. The result depends not only on the robustness of the machine learning algorithm but also on the utilized features. Bag-of-word is a common utilized features. As a statistical feature, bag-of-word does not take into account semantic of words. Previous research has indicated the potential of semantic in supervised SA task. To augment the result of sentiment analysis, this paper proposes a method to extract text features named sentence level features (SLF) and domain sensitive features (DSF) which take into account semantic of words in both sentence level and domain level of product reviews. A word sense disambiguation based method was adapted to extract SLF. For every similarity employed in generating SLF, the SentiCircle-based method was enhanced to generate DSF. Results of the experiments indicated that our proposed semantic features i.e. SLF and SLF + DSF favorably increase the performance of supervised sentiment analysis on product reviews.
\end{abstract}

Keywords: Sentiment analysis, Online product reviews, Supervised approach, Machine learning

\section{Introduction}

The exponential growth of e-commerce has triggered it to become a rich source of information nowadays. On e-commerce, customers provide a qualitative evaluation in the form of an online review that describes their opinions on a specific product [1]. With a huge number of OPRs, manual processing is not an efficient task. Sentiment analysis (SA) technique emerges in response to the requirement of processing OPRs in speed [2]. In terms of product review analysis, SA which is also named Opinion Mining can be defined as a task of recognizing customer's opinion or sentiment toward the products or the product features [3] that can be categorized into positive, negative, or neutral 
responses [4]. SA plays an important role to automate the extraction of subjective information i.e. sentiment embodied in OPRs. The success of SA application on product reviews will in turn help customers in suggesting about buying a certain product [5] based on the analysis of OPRs. Meanwhile, for companies and online marketers, they can make use SA technique to foresee customer satisfaction toward a certain product [6]. Two major approaches commonly employed for SA tasks on product reviews are lexicon-based approaches and ML-based approaches [7]. In extracting opinions or sentiments from the text data, lexicon-based methods rely on a sentiment lexicon e.g. SentiwordNet [8], SO-CAL [9], MPQA subjectivity lexicon [10], Harvard general inquirer, Bing Liu's opinion lexicon [11], SenticNet [12], and NRC emotion lexicon [13]. Sentiment lexicon is a dictionary of precompiled sentiment terms [14]. Sentiment term is term, commonly verb and adjective, representing the sentiment of the text document. In brief, lexicon-based method extract all sentiment terms for any given text and assign their sentiment value using sentiment lexicon. Meanwhile, ML-based techniques rely on ML algorithms and see SA as a regular text classification task. Text classification task assigns a piece of text data into several predefined classes involving ML algorithms [15]. In terms of SA task, ML-based techniques classify text document into one out of three classes namely positive class, neutral class, and negative class. For a given set of training text data, ML algorithms build a model based on the extracted features of a labeled text. The model is then utilized to classify unlabeled text. The result of supervised SA task is therefore influenced by the robustness of both extracted text features and ML algorithms. Mostly, recent works [16-19] dealing with supervised SA concerned more on the extension of the employed ML algorithms instead of the development of robust text features. We briefly overview those works on "Related work" section. Concerning on the extraction of text features is therefore still challenging task in the area of supervised SA.

Referring to the previously research gap, the motivation for this study comprises:

1. Enhancing the result of supervised SA by proposing a method to extract robust text features for supervised SA task.

2. Evaluating the performance of the proposed text features using several ML-algorithms and feature selection methods.

In proposing the method to extract text features for supervised SA, we consider the finding reported by [3]. Rintyarna [3] highlighted the importance of semantics for SA task. Taking into account semantics of words is important for SA since the same term appears in different text data may reveals different meaning i.e. different sentiment value. In turn, capturing sementics is potential to augment the result of Sentiment Analysis task. In this study, we present a method to extract text features capturing semantic in sentence level and domain level of product reviews. We introduce two feature sets namely sentence level feature (SLF) and domain sensitive feature (DSF). For extracting SLF, a WSD based technique was adapted [20]. And for extracting DSF, a Senti-Circle based method was enhanced. We arrange several scenarios of experiment using several ML algorithms and feature selection methods to evaluate our proposed features compared with common features employed for SA task i.e. BOW. We utilized Waikato Environment for Knowledge Analysis (WEKA) for the implementation of ML-algorithms 
and feature selection methods. The result of experiment indicated that our proposed features outperformed BOW.

The rest of the manuscript is arranged in the following sections. "Related work" section reviews state of the art study related with this work. "Proposed method" section describes the proposed method for extracting SLF and DLF. We explore the result of experiment and the discussion in "Experimental results and discussion" section. Finally we summarize the result of this work in "Conclusion" section.

\section{Related work}

Using BOW, [16] performed an SA task on an Amazon product review dataset. RFSVM, a hybrid method that combines Random Forest (RF) and Support Vector Machine (SVM), was employed to make use of the capabilities of both classifiers. Precision, recall, F-Measure, and accuracy were used as the performance metrics to evaluate the proposed method compared with the baseline methods i.e. RF and SVM. Using instances of 500 positive datasets and 500 negative datasets, the result of the experiment showed that RFSVM outperformed the baseline methods in terms of all three performance metrics.

A word embedding-based sentiment classification is proposed [17]. Using google toolkit word2vec, a continuous bag-of-words (CBOW) model and a Skip-gram model were generated in order to produce meaningful features. For representing the document, the sum of weighted word embeddings was used. Combined with SVM, this work proposed an extension of the SVM classifier, called SVM-WE. The method was evaluated using four datasets i.e. RT-s, CR, RT-2k, and IDBM. The result of the experiment indicated that the proposed method performed slightly better compared with the baseline method.

Another work [18] proposed a set of 13 sentiment features for supervised SA in Twitter dataset classification. Features F1 to F8 were generated based on three sentiment lexicons, i.e. SenticNet, SentiWordNet, and NRC Emotion Lexicon. Features F9 to F13 were generated using a seed word list i.e. Subjective Words. Two datasets, namely TaskA Twitter and TaskB Twitter, were employed to validate feature performance in classification. The Naïve Bayes classifier was used as performance metric to calculate its accuracy. The best accuracy achieved by the proposed features was $75.60 \%$.

In order to analyze social media content, Yoo [19] proposed a system to predict user sentiment. For representing the text data, the work adopted a two-dimensional representation of word2vec. The model for the sentiment analysis task was built using Convolutional Neural Network for Sentence Classification (CNN) by making use of TensorFlow, an open-source library for various dataflow programming tasks. Validated using the Sentiment140 dataset, containing 800,000 positive documents and 800,000 negative documents, the proposed model outperformed th baseline method i.e. Naïve Bayes, SVM, and Random Forest.

As an utmost advanced topic in the field of Natural Language Processing (NLP), many approaches have been developed for SA application [21]. Among the approaches is called Aspect Based Sentiment Analysis (ABSA). The main task of ABSA is inferring the sentiment polarity toward a specific target called aspect within a given piece of text data. In terms of product review analysis, it is useful for 
determining the product features that require improvement [22]. In the following paragraphs we briefly review several works discussing ABSA.

A method called joint aspect-based sentiment topic (JABST) has been introduced [23]. It proposed a unified framework to perform common ABSA task including aspect extraction and sentiment polarity identification. The study made use graphical model to describe relationship among aspects, opinion, sentiment polarity and granularity. A maximum entropy based model called MaxEnt-JABST has also been proposed to improve the word distribution description. In the evaluation step, two real world datasets from [24] were employed. The evaluation step focused on two points i.e.: (1) comparing the quality of the extracted topics and (2) calculating the precision of aspects and opinions. The results of experiment confirmed that the JABST significantly outperformed baseline model.

To perform ABSA tasks on customer reviews, a novel system called W2VLDA was presented by [25] based on the combination of a topic modeling approach and a Maximum Entropy classifier. The system performed the main tasks of ABSA simultaneously. Employing Brown cluster to train the model of Maximum Entropy classifier, W2VLDA was able to separate aspect-terms and opinion-words into word classes without any language dictionary. The work conducted experiment to evaluate the performance of different subtasks using different datasets. Restaurant review dataset [26] containing domain-related aspects was used to evaluate aspect category classification. Dataset on the domain of Laptops and Digital-SLR [24] containing English reviews was employed to evaluate sentiment classification subtask. Meanwhile, SemEval-2016 task 5 from [27] was used to perform multilingual experiments. Compared with the other LDA-based approaches as baseline methods, the system achieved slightly better results.

Another work [28] focused on three subtasks of ABSA i.e.: sentiment extraction, aspect assignment, and aspect category determination. The work contributed to improving the functionality of the current state-of-the-art topic model approach by adding product description as another dimension of the model. Two extended topic model-based ABSA methods were presented: Seller-aided Aspect-based Sentiment Model (SA-ASM) and Seller-aided Product-based Sentiment Model (SA-PSM). SAASM outperformed two baseline methods on sentiment classification and aspect assignment. Meanwhile, SA-PSM performed better compared with the baseline methods on subtask aspect categorization.

Aspect extraction which aims at identifying the object of user's opinion from online reviews holds an important role in ABSA approach. Motivated by the vulnerability of syntactic patterns-based approach due to its dependency to dependency parser, a study [29] proposed two-fold rule-based model (TF-RBM) to perform ABSA tasks. Sequential pattern-based rules (SPR) [30] was firstly employed to extract all aspects and opinions. Since many extracted aspects were not related to the product, the study performed a pruning method based on normalized Google distance calculation to improve aspect extraction accuracy. The last step of the proposed method was called concept extraction i.e. domain specific opinions that reveal user's sentiment. 


\section{Proposed method}

The steps of the proposed method are: (1) capturing semantic values in product review texts at the sentence level and extracting the sentence level features (SLF), (2) capturing semantic values in product reviews influenced by different product domain extracting the domain sensitive features (DSF). Since there are many notations employed in this section, we present details of the notations in Table 1.

\section{Extracting sentence level feature (SLF)}

Capturing sentence-level semantic is important since the same words that appear in different piece of text may share different meaning i.e. different sentiment value as described in Table 1. In Table 2, we describe that the word "enjoy" has different sense i.e. different sentiment value when it appears in different sentence. This characteristic is known as polysemy. The task aims at assigning correct sentiment value to a word with respect to its local context i.e. sentence. We describe the step of extracting SLF in Fig. 1.

Table 1 Details of notations

\begin{tabular}{|c|c|}
\hline Notations & Details \\
\hline$D$ & Product review document \\
\hline$S_{k}$ & Review sentence within $D$ with index-k \\
\hline$w_{i}$ & Word with index- $i$ \\
\hline$w s_{i}^{j}$ & Sense of word $i$ with index- $j$ \\
\hline spos $_{i}^{j}$ & Raw positive sentiment value of $w s_{i}^{j}$ picked from SentiwordNet \\
\hline sneg $j_{i}^{j}$ & Raw negative sentiment value of $w_{i}^{j}$ picked from SentiwordNet \\
\hline sneut $_{i}^{j}$ & Raw neutral sentiment value of $w s_{i}^{j}$ picked from SentiwordNet \\
\hline $\operatorname{sim}_{a b}^{c d}$ & $\begin{array}{l}\text { Similarity value between } w s_{a}^{c} \text { and } w s_{b}^{d} \text { calculated using one of } \\
\text { Wordnet similarity algorithms }\end{array}$ \\
\hline $\operatorname{deg}\left(w s_{i}^{j}\right)$ & Indegree score of $w s_{i}^{j}$ \\
\hline csposi $_{i}$ & Contextual positive sentiment value of $w_{i}$ \\
\hline csneg $_{i}$ & Contextual negative sentiment value of $w_{i}$ \\
\hline csneu $_{i}$ & Contextual neutral sentiment value of $w_{i}$ \\
\hline fpos $S_{k}$ & Positive value of feature of $S_{k}$ \\
\hline fnegs $_{k}$ & Negative value of feature of $S_{k}$ \\
\hline fneus $_{k}$ & Neutral value of feature of $S_{k}$ \\
\hline fpos $D$ & Positive value of feature of $D$ \\
\hline fnegD & Negative value of feature of $D$ \\
\hline fneud & Neutral value of feature of $D$ \\
\hline wd & Domain word of product review dataset \\
\hline$p w_{k}$ & Pivot word of review sentence $S_{k}$ \\
\hline$\theta_{i}$ & Angle representing semantic orientation adjustment of $w_{i}$ \\
\hline$r_{i}$ & Degree of correlation between $p w_{k}$ and $w_{i}$ \\
\hline$c t s_{i}$ & Prior sentiment value of $w_{i}$ determined using Rule (11) \\
\hline$x_{i}$ & Senticircle representation in Cartesian coordinate \\
\hline$y_{i}$ & Senticircle representation in Cartesian coordinate \\
\hline$f \times S_{k}$ & Feature value of $S_{k}$ calculated using $x$ of Senticircle \\
\hline fy $S_{k}$ & Feature value of $S_{k}$ calculated using y of Seinticircle \\
\hline$f \times D$ & Feature value of $D$ calculated using $x$ of Senticircle \\
\hline fyd & Feature value of $D$ calculated using y of Senticircle \\
\hline
\end{tabular}


Table 2 Example of different sentiment of the word "enjoy"

\begin{tabular}{llll}
\hline Word & Sentence & Sense & Sentiment \\
\hline enjoy & $\begin{array}{l}\text { I enjoy using the camera of this smartphone } \\
\text { The vendor enjoys new regulation issued by the } \\
\text { authority }\end{array}$ & $\begin{array}{l}\text { Get pleasure from } \\
\text { Possess and benefit from }\end{array}$ & Positive \\
& & Neutral \\
\hline
\end{tabular}

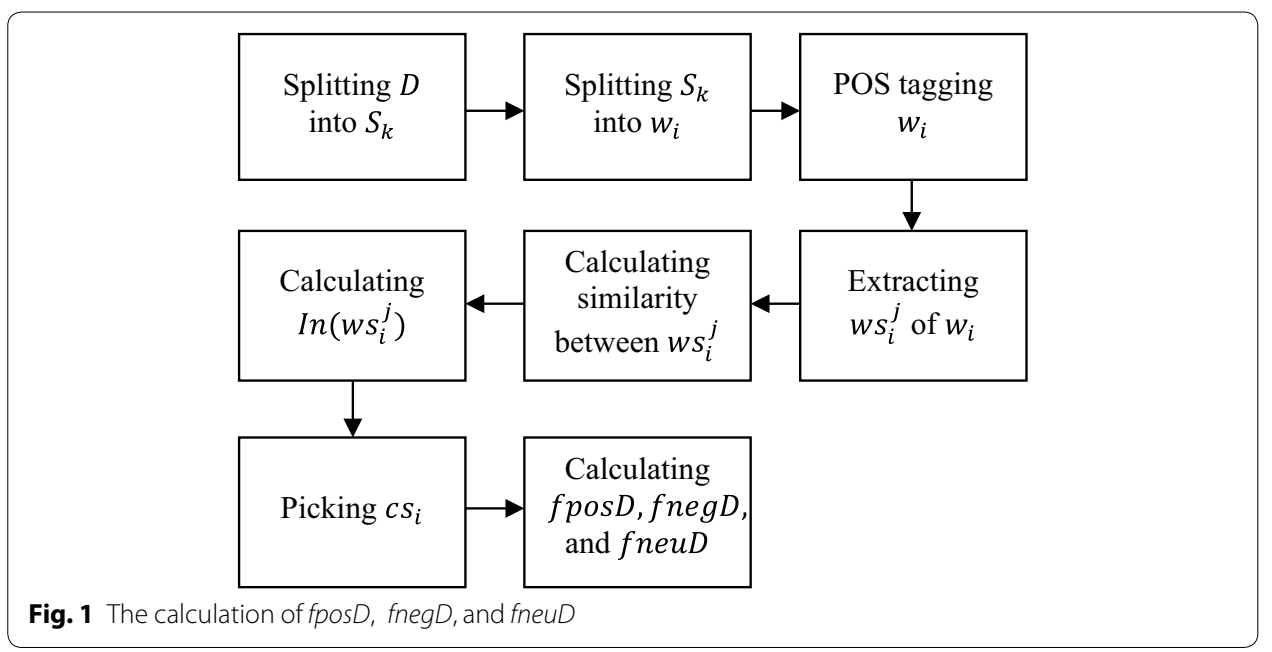

To capture semantic value in product reviews at sentence level i.e. extracting SLF, product review document $D$ is split into review sentence $S_{k}$. The process is done at sentence level. Suppose $S_{k}$ consists of $n$ words, $w_{1}, w_{2}, \ldots w_{n}$. The aim of this stage is to find contextual sentiment value $c s_{i}$ of word $w_{i}$ associated with sentiment score $s_{i}$ picked from SentiwordNet [8]. In the next step, part of speech (POS) tagging is done, which is part of common text processing, including filtering. It is a process of assigning a part of speech value to a word in a piece of text [31]. Since we employ SentiwordNet [8], which is based on WordNet [32], POS tagging is important for selecting the correct sense of $w_{i}$ in accordance with its POS tag [33]. WordNet [32] itself employs 4 POS tags, i.e. noun, verb, adjective, and adverb. POS tagging is important for the next step, i.e. extracting $w s_{i}^{j}$ from $w_{i}$. For every extracted $w s_{i}^{j}$ its associated sentiment value is picked from SentiwordNet [8]. Every $w s_{i}^{j}$ has three different sentiment scores, namely $\operatorname{spos}_{i}^{j}$, sneg ${ }_{i}^{j}$, and sneu $_{i}^{j}$.

The similarity between $w s_{i}^{j}$ is calculated using WordNet similarity algorithms, i.e. from Lin, Jiang and Conrath, Resnik, Leacock and Chodorow, and Wu and Palmer. Adapted Lesk [34] is also employed. Similarity between word senses, denoted as $\operatorname{sim}_{a b}^{c d}$, means similarity value of $w s_{a}^{c}$ and $w s_{b}^{d}$. They are calculated for all possible combinations, as can be seen in Table 3 . The calculation adopts the WSD technique firstly introduced by [20]. For simple, the task illustrated in Table 4 can be assumed as building undirected weighted graph of every review sentence with $w s_{i}^{j}$ as the vertex and $\operatorname{sim}_{a b}^{c d}$ as the weight of the edges of the graph.

The results of the previous step are the three different sentiment scores from SentiWordNet [8]. For example, the result of processing the review sentence 'The screen is 
Table 3 Similarity between word senses

\begin{tabular}{|c|c|c|c|c|c|c|}
\hline & & \multicolumn{3}{|l|}{$w_{1}$} & \multicolumn{2}{|l|}{$w_{2}$} \\
\hline & & $w s_{1}^{1}$ & $w s_{1}^{2}$ & $w s_{1}^{3}$ & $w s_{2}^{1}$ & $w s_{2}^{2}$ \\
\hline \multirow[t]{3}{*}{$w_{1}$} & $w s_{1}^{1}$ & & & & $\operatorname{sim}_{12}^{11}$ & $\operatorname{sim}_{12}^{12}$ \\
\hline & $w s_{1}^{2}$ & & & & $\operatorname{sim}_{12}^{21}$ & $\operatorname{sim}_{12}^{22}$ \\
\hline & $w s_{1}^{3}$ & & & & $\operatorname{sim}_{12}^{31}$ & $\operatorname{sim}_{12}^{32}$ \\
\hline \multirow[t]{2}{*}{$w_{2}$} & $w s_{2}^{1}$ & & & & & \\
\hline & $w s_{2}^{2}$ & & & & & \\
\hline
\end{tabular}

Table 4 Word senses along with their sentiment score

\begin{tabular}{|c|c|c|c|}
\hline Word & Senses & & Sentiment score \\
\hline \multicolumn{4}{|l|}{$W_{1}$} \\
\hline \multirow[t]{9}{*}{ Screen } & \multirow[t]{3}{*}{$w_{1}^{1}$} & \multirow{3}{*}{$\begin{array}{l}\text { A white or silvered surface where pictures can be } \\
\text { projected for viewing }\end{array}$} & $\operatorname{spos}_{1}^{1}$ \\
\hline & & & sneg $_{1}^{1}$ \\
\hline & & & sneut \\
\hline & \multirow[t]{3}{*}{$w_{1}^{2}$} & \multirow{3}{*}{$\begin{array}{l}\text { A protective covering that keeps things out or hinders } \\
\text { sight }\end{array}$} & $\operatorname{spos}_{1}^{2}$ \\
\hline & & & sneg ${ }_{1}^{2}$ \\
\hline & & & $\operatorname{sneu}_{1}^{2}$ \\
\hline & \multirow[t]{3}{*}{$w_{1}^{3}$} & \multirow[t]{3}{*}{ The personnel of the film industry } & $\operatorname{spos}_{1}^{3}$ \\
\hline & & & sneg ${ }_{1}^{3}$ \\
\hline & & & $\operatorname{sneu}_{1}^{3}$ \\
\hline \multicolumn{4}{|l|}{$w_{2}$} \\
\hline \multirow[t]{6}{*}{ Great } & \multirow[t]{3}{*}{$w_{2}^{1}$} & \multirow[t]{3}{*}{ Relatively large in size or number or extent } & $\operatorname{spos}_{2}^{1}$ \\
\hline & & & sneg ${ }_{2}^{1}$ \\
\hline & & & sneu $_{2}^{?}$ \\
\hline & \multirow[t]{3}{*}{$w_{2}^{2}$} & \multirow[t]{3}{*}{ Of major significance or importance } & $\operatorname{spos}_{2}^{2}$ \\
\hline & & & sneg $_{2}^{2}$ \\
\hline & & & $\operatorname{sneu}_{2}^{2}$ \\
\hline
\end{tabular}

great', can be seen in Table 4. After the POS tagging step, including filtering, there are two words, i.e. 'screen' with POS tag noun and 'great' with POS tag adjective.

To assign $c s_{i}$ of $w_{i}$, the indegree score of $w s_{i}^{j}$, denoted by $\operatorname{In}\left(w s_{i}^{j}\right)$, is calculated. Indegree score is important to assign contextual sense of $w_{i}$. Among the senses of $w_{i}$ i.e. $w s_{i}^{j}$, a sense with the highest Indegree score is assigned as contextual sense of $w_{i}$. Contextual sense is a sense where $\operatorname{cspos}_{i}$, csneg $_{i}$, and csneu $_{i}$ are picked from the collection of SentiwordNet and assigned as contextual sentiment value of $w_{i}$. For the above case there are three indegree scores for $w_{1}$, i.e. $\operatorname{deg}\left(w s_{1}^{1}\right), \operatorname{deg}\left(w s_{1}^{2}\right)$ and $\operatorname{deg}\left(w s_{1}^{3}\right)$ while there are two indegree scores for $w_{2}$, i.e. $\operatorname{deg}\left(w s_{2}^{1}\right)$ and $\operatorname{deg}\left(w s_{2}^{2}\right)$. They are calculated as follows:

$$
\begin{aligned}
& \operatorname{deg}\left(w s_{1}^{1}\right)=\operatorname{sim}_{12}^{11}+\operatorname{sim}_{12}^{12} \\
& \operatorname{deg}\left(w s_{1}^{2}\right)=\operatorname{sim}_{12}^{21}+\operatorname{sim}_{12}^{22} \\
& \operatorname{deg}\left(w s_{1}^{3}\right)=\operatorname{sim}_{12}^{31}+\operatorname{sim}_{12}^{32} .
\end{aligned}
$$


The next task is determining the selected sense of $w_{i}$ by calculating $\max \left\{\operatorname{deg}\left(w s_{1}^{1}\right), \operatorname{deg}\left(w s_{1}^{2}\right), \operatorname{deg}\left(w s_{1}^{3}\right)\right\}$. The sense that has the highest indegree score is selected as the contextual sense of $w_{i}$ and its sentiment score is labeled with csposi, csneg $_{i}$, or csneu $_{i}$. Once these values have been assigned for every $w_{i}$, the last procedure in this step is calculating the numeric feature value at the sentence level, fpos $S_{k}$, fnegS $S_{k}$, and fneu $S_{k}$, using Eqs. (1), (2) and (3).

$$
\begin{aligned}
& \text { fpos }_{k}=\sum_{i=1}^{n} \text { cspos }_{i} \\
& \text { fnegS }_{k}=\sum_{i=1}^{n} \text { cspos }_{i} \\
& \text { fneu }_{k}=\sum_{i=1}^{n} \operatorname{cspos}_{i}
\end{aligned}
$$

where $n$ is the number of words in $S_{k}$. To calculate the numeric feature value at review document level, Eqs. (4), (5), and (6) are employed. For $o$ is the number of sentences in review document $D, f p o s D$, fnegD, and fneuD are calculated as follows:

$$
\begin{aligned}
& \text { fpos } D=\frac{\sum_{k=1}^{o} f \text { pos } S_{k}}{k} \\
& f \text { negD }=\frac{\sum_{k=1}^{o} f \text { negS }}{k} \\
& \text { fneuD }=\frac{\sum_{k=1}^{o} \text { fneu }_{k}}{k}
\end{aligned}
$$

\section{Capturing domain sensitive features (DSF)}

In this step, we adopt Senticircle approach [35]. The main principle of Senticircle suggest that terms exist in the same context tend to share the same semantics. In terms of product review, we define the context as product domain. In consequence, the same terms that appears in different product domains tend to share different meaning. In terms of SA, sharing different meanings means carrying different sentiment. For example, 'long battery life' in Electronics domain express positive sentiment, while 'long stopping time' in the Automobile domain share negative sentiment.

$$
\begin{aligned}
& \operatorname{maxSim}=\operatorname{argmax}_{\operatorname{Sim}_{i}} \operatorname{Sim}_{i}\left(w d, w_{i}\right) \\
& \operatorname{Sim}_{i}=\frac{2 * \operatorname{Depth}\left(L C S\left(w d, w_{i}\right)\right)}{\operatorname{Depth}(w d)+\operatorname{Depth}\left(w_{i}\right)} .
\end{aligned}
$$

To generate the DSF, several formulas are provided. Figure 2 describes the steps that need to be carried out. The first three steps, including POS tagging, are the same as in the first step of the method. The next step is determining pivot word $p w_{k}$ of sentence $S_{k}$. 


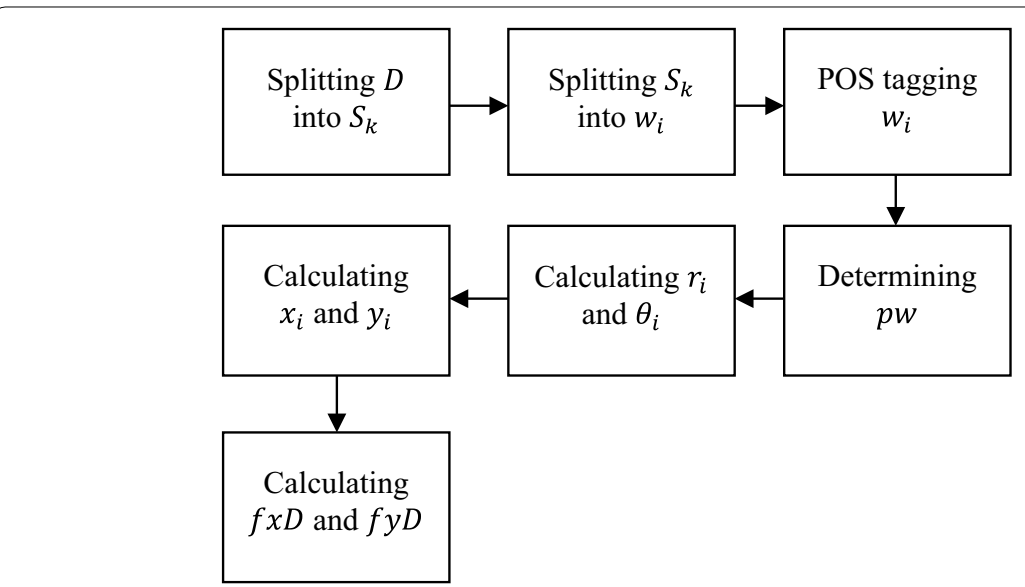

Fig. 2 The calculation of $f x D$ and $f y D$

A pivot word is a representative of the domain word at sentence level [3]. In this work, $p w_{k}$ is defined as the noun with the closest similarity to the domain word. For measuring similarity, Wu and Palmer's algorithm is employed [36]. For $w d$ as the domain word (e.g. Smartphone, Book, Beauty, or Computers), the similarity between $w d$ and $w_{i}$ is computed using (7) and (8). The pivot word from $w_{i}$ that has the highest value, maxSim, is selected.

In Eq. (8), LCS means the Least Common Subsumer between the first sense of $w d$ and the contextual sense of $w_{i}$ in the WordNet [32] taxonomy. Since the method from [37] was adopted in this stage, $r_{i}$ is computed to represent the distance between $w_{i}$ and $p w_{k}$ using Eq. (9). In (9), $N$ is the total number of words in the corpus of product reviews and $N w_{i}$ is the total number of $w_{i}$.

$$
r_{i}=f\left(p w_{k}, w_{i}\right) \log \frac{N}{N w_{i}}
$$

To generate the SentiCircle representation of $w_{i}$, we need to assign $\theta_{i}$ using Eq. (10).

$$
\theta_{i}=c t s_{i} * \pi \mathrm{rad}
$$

In Eq. (10), cts $s_{i}$ is determined using rule (11).

$$
\text { cts }_{i}=\left\{\begin{array}{l}
\text { cspos }_{i} \text { if } \mid \text { cspos }_{i}|>| \text { csneg }_{i} \mid \\
\text { csneg }_{i} \text { if } \mid \text { csneg }_{i}|>| \text { cspos }_{i} \mid
\end{array}\right.
$$

The last step is to generate the SentiCircle representation by using (12) and (13). The sentiment value of a word is represented using the values of $x$ and $y$ in a Cartesian coordinate system as seen in Fig. 3. To calculate the numeric value of the features in sentence $S_{k}$, Eqs. (14) and (15) are introduced, where $N w S_{k}$ is the number of words in $S_{k}$.

$$
\begin{aligned}
& x_{i}=r_{i} \cos \theta_{i} \\
& y_{i}=r_{i} \sin \theta_{i} \\
& f x S_{k}=\frac{\sum_{i=1}^{N w S_{k}} x_{i}}{N w S_{k}}
\end{aligned}
$$




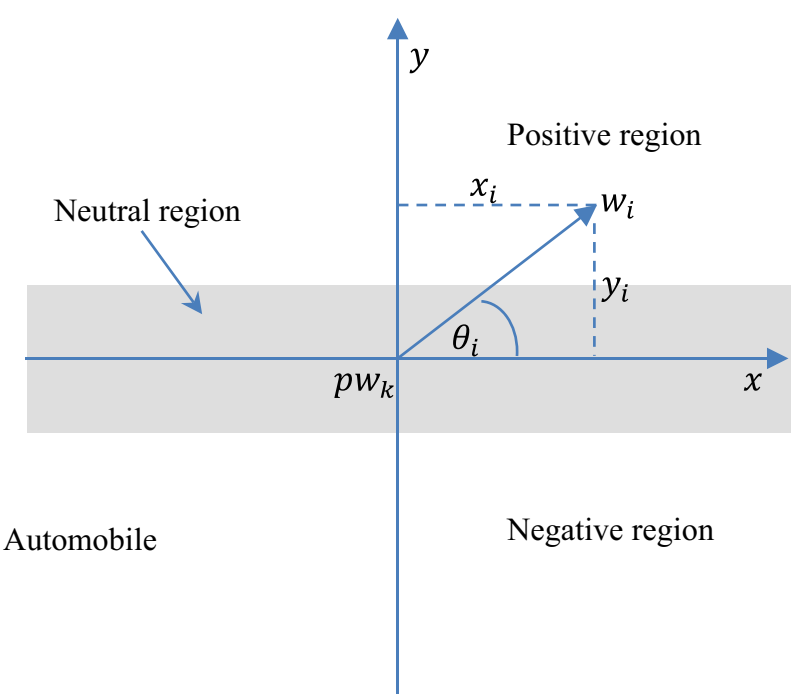

Fig. 3 Representation of Senticircle in Cartesian coordinate system

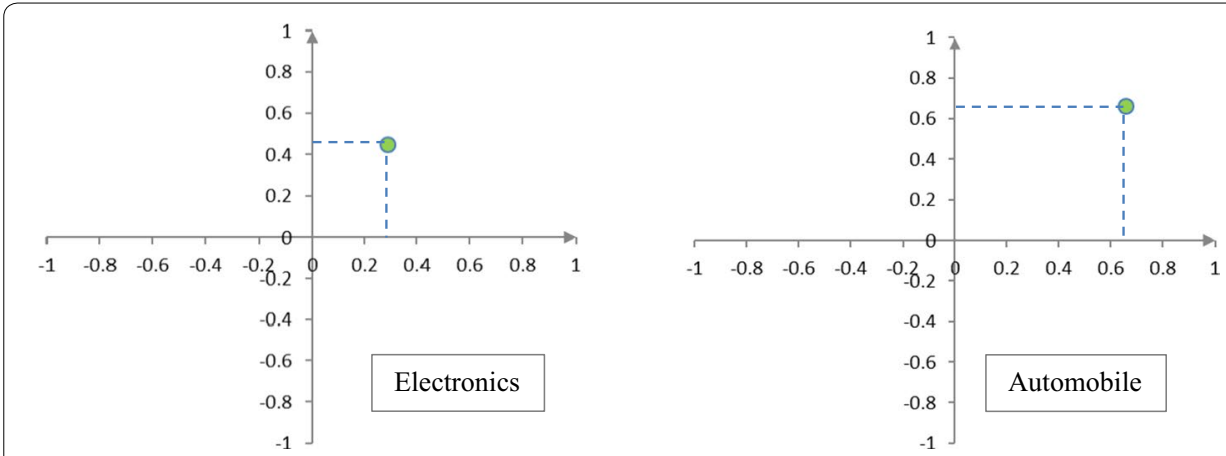

Fig. 4 Example of how Senticircle adjust Sentiment value of the word "Iong" of two different domains

$$
f y S_{k}=\frac{\sum_{i=1}^{N w S_{k}} y_{i}}{N w S_{k}}
$$

In Fig. 4, we provide an example of how Senticircle adjust a sentiment value of the same word "long" but from different domain e.g. Electronics and Automobile. The word "long" is picked from review document of the dataset as presented in Table 5. In Table 5, we also provide the variable value of the Senticircle of the word "long". In the first domain e.g. Electronics, the word "long" has relatively neutral value while in the second domain e.g. Automobile, this word has highly positive value. The value of $x_{i}$ and $y_{i}$ presented in the table is the value after normalization.

To represent a document with its semantic features, the numeric value of the features in the review document is calculated using Eqs. (16) and (17). In both equations, $o$ is the number of sentences in $D$. For every similarity algorithm, a set of features is generated, 
Table 5 Variable value of the word "long" calculated for both domains

\begin{tabular}{|c|c|c|}
\hline \multirow[t]{2}{*}{ Variable value } & \multicolumn{2}{|l|}{ Domain } \\
\hline & Electronics & Automobile \\
\hline Review sentence & $\begin{array}{l}\text { I mounted a shelf above the TV to get the } \\
\text { cable box out of the way and avoid having } \\
\text { to run a long HDMI cable through the wall }\end{array}$ & $\begin{array}{l}\text {... but they are built solid, nice tough big hard } \\
\text { clamps and love having a long cable so I } \\
\text { never have to move cars around or anything } \\
\text { if needed }\end{array}$ \\
\hline$p w_{k}$ & 230 & 495 \\
\hline$f\left(p w_{k}, w_{i}\right)$ & 85 & 145 \\
\hline$N$ & 130,765 & 170,873 \\
\hline$N w_{i}$ & 186 & 208 \\
\hline$r$ & 241 & 422 \\
\hline $\mathrm{Cts}_{i}$ & 0.32 & 0.25 \\
\hline$\theta_{i}$ & $57.6^{\circ}$ & $45^{\circ}$ \\
\hline$x_{i}$ & 0.29 & 0.66 \\
\hline$y_{i}$ & 0.45 & 0.66 \\
\hline
\end{tabular}

Italic values indicate Senticircle parameters calculated in both domains

i.e.: fpos $D$, fnegD, fneuD, $f x D$, and $f y D$. Since 5 similarity algorithms are employed (Wu and Palmer, Jiang and Conrath, Leacock and Chodorow, Resnik, and Li), the complete set of review document features consists of 25 features, as listed in Table 6. In the table, we describe the notation of the features, the details and the type of the features. F1-F15 is local features. Meanwhile, F16-F25 is domain sensitive features.

$$
\begin{gathered}
f x D=\frac{\sum_{k=1}^{o} S_{k}}{o} \\
f y D=\frac{\sum_{k=1}^{o} S_{k}}{o}
\end{gathered}
$$

\section{Experimental results and discussion}

\section{Experimental setup}

An experiment was conducted to evaluate the features extracted by the proposed method employing several machine learning algorithms available in WEKA [38], i.e. Bayesian Network, Naïve Bayes, Naïve Bayes Multinomial, Logistic, Multilayer Perceptron, J48, Random forest, and Random tree. Another experiment was conducted using feature selection method. In the implementation, WEKA feature selection methods were employed, i.e.: ClassifierAttributeEval (CA), GainRatioAttributeEval (GR), InfoGainAttributeEval, OneRAttributeEval (OneR) and PrincipalComponent (PCA). Precision, recall and F-measure were calculated as performance metrics. Although important, extending Machine learning algorithms is not part of our contribution. A key point of this work is to demonstrate as well as to evaluate the performance of our proposed semantic features. For that reason, in all experiment we employ default setting of the ML parameters provided by WEKA to avoid bias in the result of experiment. The experiments were performed on IBM System X3400 M3 Tower Server. 
Table 6 Details of the features

\begin{tabular}{|c|c|c|}
\hline Feature & Details & Type \\
\hline \multicolumn{3}{|l|}{ F1 } \\
\hline fposD(wup) & $\begin{array}{l}\text { Average positive value of review document where Wu and } \\
\text { Palmer is employed as similarity algorithm }\end{array}$ & Sentence level features (SLF) \\
\hline \multicolumn{3}{|l|}{ F2 } \\
\hline fnegD(wup) & $\begin{array}{l}\text { Average negative value of review document where Wu and } \\
\text { Palmer is employed as similarity algorithm }\end{array}$ & \\
\hline \multicolumn{3}{|l|}{ F3 } \\
\hline fneuD(wup) & $\begin{array}{l}\text { Average neutral value of review document where Wu and } \\
\text { Palmer is employed as similarity algorithm }\end{array}$ & \\
\hline \multicolumn{3}{|l|}{$\mathrm{F} 4$} \\
\hline$f p o s D(j c n)$ & $\begin{array}{l}\text { Average positive value of review document where Jiang and } \\
\text { Conrath is employed as similarity algorithm }\end{array}$ & \\
\hline \multicolumn{3}{|l|}{ F5 } \\
\hline fnegD $(j c n)$ & $\begin{array}{l}\text { Average negative value of review document where Jiang and } \\
\text { Conrath is employed as similarity algorithm }\end{array}$ & \\
\hline \multicolumn{3}{|l|}{ F6 } \\
\hline fneuD(jcn) & $\begin{array}{l}\text { Average neutral value of review document where Jiang and } \\
\text { Conrath is employed as similarity algorithm }\end{array}$ & \\
\hline \multicolumn{3}{|l|}{ F7 } \\
\hline fposD(lch) & $\begin{array}{l}\text { Average positive value of review document where Leacock and } \\
\text { Chodorow is employed as similarity algorithm }\end{array}$ & \\
\hline \multicolumn{3}{|l|}{ F8 } \\
\hline fnegD (Ich) & $\begin{array}{l}\text { Average negative value of review document where Leacock and } \\
\text { Chodorow is employed as similarity algorithm }\end{array}$ & \\
\hline \multicolumn{3}{|l|}{ F9 } \\
\hline fneuD(Ich) & $\begin{array}{l}\text { Average neutral value of review document where Leacock and } \\
\text { Chodorow is employed as similarity algorithm }\end{array}$ & \\
\hline \multicolumn{3}{|l|}{ F10 } \\
\hline fpos $D(r e s)$ & $\begin{array}{l}\text { Average positive value of review document where Resnik is } \\
\text { employed as similarity algorithm }\end{array}$ & \\
\hline \multicolumn{3}{|l|}{ F11 } \\
\hline fnegD(res) & $\begin{array}{l}\text { Average negative value of review document where Resnik is } \\
\text { employed as similarity algorithm }\end{array}$ & \\
\hline \multicolumn{3}{|l|}{$\mathrm{F} 12$} \\
\hline fneuD(res) & $\begin{array}{l}\text { Average neutral value of review document where Resnik is } \\
\text { employed as similarity algorithm }\end{array}$ & \\
\hline \multicolumn{3}{|l|}{ F13 } \\
\hline fposD $($ lin $)$ & $\begin{array}{l}\text { Average positive value of review document where Lin is } \\
\text { employed as similarity algorithm }\end{array}$ & \\
\hline \multicolumn{3}{|l|}{ F14 } \\
\hline fnegD(lin) & $\begin{array}{l}\text { Average negative value of review document where Lin is } \\
\text { employed as similarity algorithm }\end{array}$ & \\
\hline \multicolumn{3}{|l|}{ F15 } \\
\hline fneuD(lin) & $\begin{array}{l}\text { Average neutral value of review document where Lin is } \\
\text { employed as similarity algorithm }\end{array}$ & \\
\hline F16 & & \\
\hline
\end{tabular}


Table 6 (continued)

\begin{tabular}{|c|c|c|}
\hline Feature & Details & Type \\
\hline$f \times D(w u p)$ & $\begin{array}{l}\text { Average } x \text { value of review document where Wu and Palmer is } \\
\text { employed as similarity algorithm }\end{array}$ & Domain sensitive features (DSF) \\
\hline \multicolumn{3}{|l|}{ F17 } \\
\hline fyD(wup) & $\begin{array}{l}\text { Average } y \text { value of review document where Wu and Palmer is } \\
\text { employed as similarity algorithm }\end{array}$ & \\
\hline \multicolumn{3}{|l|}{ F18 } \\
\hline$f \times D(j c n)$ & $\begin{array}{l}\text { Average } x \text { value of review document where Jiang and Conrath } \\
\text { is employed as similarity algorithm }\end{array}$ & \\
\hline \multicolumn{3}{|l|}{ F19 } \\
\hline fyD (jcn) & $\begin{array}{l}\text { Average y value of review document where Jiang and Conrath } \\
\text { is employed as similarity algorithm }\end{array}$ & \\
\hline \multicolumn{3}{|l|}{ F20 } \\
\hline$f \times D(I c h)$ & $\begin{array}{l}\text { Average } x \text { value of review document where Leacock and Cho- } \\
\text { dorow is employed as similarity algorithm }\end{array}$ & \\
\hline \multicolumn{3}{|l|}{ F21 } \\
\hline fyD $(I c h)$ & $\begin{array}{l}\text { Average } y \text { value of review document where Leacock and Cho- } \\
\text { dorow is employed as similarity algorithm }\end{array}$ & \\
\hline \multicolumn{3}{|l|}{$\mathrm{F} 22$} \\
\hline$f \times D(r e s)$ & $\begin{array}{l}\text { Average } x \text { value of review document where Resnik is employed } \\
\text { as similarity algorithm }\end{array}$ & \\
\hline \multicolumn{3}{|l|}{ F23 } \\
\hline fyD (res) & $\begin{array}{l}\text { Average y value of review document where Resnik is employed } \\
\text { as similarity algorithm }\end{array}$ & \\
\hline \multicolumn{3}{|l|}{ F24 } \\
\hline$f \times D(\operatorname{lin})$ & $\begin{array}{l}\text { Average } x \text { value of review document where Lin is employed as } \\
\text { similarity algorithm }\end{array}$ & \\
\hline \multicolumn{3}{|l|}{ F25 } \\
\hline fyD $($ lin $)$ & $\begin{array}{l}\text { Average y value of review document where Lin is employed as } \\
\text { similarity algorithm }\end{array}$ & \\
\hline
\end{tabular}

\section{Dataset description}

The experiment was conducted using Amazon product data [39] downloaded from http://jmcauley.ucsd.edu/data/amazon/. The collection contains product review dataset grabbed from Amazon including 142.8 millions reviews. The experiment was conducted on a small subset of this collection, i.e. the electronics and automobile datasets. The number of sample for building model and running evaluation follow the rule of tenfold cross-validation. The dataset contains reviewerID, asin, reviewerName, helpfulness, reviewText, overall, summary, unixReviewTime, and reviewTime as described in Table 7. We pick the review text for experiment from reviewText. To build the ground truth, we established a label out of three sentiment categories i.e. positive, negative, and neutral for every reviewText based on its overall score. Datasets with overall score 1-2 were assigned as negative reviews. Meanwhile, reviewTexts with overall score 4-5 were labeled positive. And the rest was assigned as neutral review. 
Table 7 Dataset details

\begin{tabular}{ll}
\hline Data & Details \\
\hline reviewerID & ID of the reviewer \\
asin & ID of the product \\
revewerName & Name of the reviewer \\
Helpfulness & Helpfulness rating of the review \\
reviewText & Text of the review \\
Overall & Rating of the product \\
Summary & Summary of the review \\
unixReviewTime & Time of the review (unix time) \\
reviewTime & Time of the review (raw time) \\
\hline
\end{tabular}

\section{Results and discussion}

Three scenarios were arranged for the experiment, i.e. (1) using a baseline features i.e. BoW (BF) that is commonle employed for recent supervised sentiment analysis task, (2) using sentence level feature only (SLF), and (3) using sentence level features combined with domain sensitive features (SLF + DSF). For each scenario, we calculate precision, recall and F-measures as the performance metrics in tenfold cross validation. We present the result of the experiment in Tables 8 and 9.

We reveal the result of experiment using Electronic dataset on Table 8. We indicate the best performance of both SLF and SLF + DSF for precision, recall and F-measure using asterisk symbol. The best performance of SLF for precision, recall, and $\mathrm{f}$ measure is $0.792,0.817$, and 0.758 respectively. Meanwhile, SLF + DSF achieve the best performance by $0.823,0.800$, and 0.760 for precision, recall and F-measure respectively.

In Table 9, we describe the result of experiment using Automobile dataset. We also indicate the best performance of SLF and SLF + DSF using asterisk symbol. The top performance of SLF for Automobile dataset is achieved for precision, recall, and F-measure by $0.796,0.847$, and 0.811 respectively. Meanwhile, SLF + DSF works best for precision, recall, and F-measure by $0.825,0.854$, and 0.831 respectively.

In Fig. 5, we calculate the average performance of our proposed features over all ML algorithms and feature selection methods compared with the baseline features. We present the result in the bar charts. Both bar charts indicate that our proposed features outperformed the baseline features measured in all performance metrics. In average SLF favorably increase the performance by $6.2 \%, 6.1 \%$, and $6.0 \%$ for precision, recall, and F-measure respectively. Meanwhile, SLF + DSF successfully augment the performance by $7.1 \%, 7.2 \%$, and $7.4 \%$ for precision, recall and F-measure. Overall trend, SLF + DSF is better than SLF by $0.8 \%, 1 \%$, and $1.2 \%$ for precision, recall and F-measure. Yet, in Electronic dataset, SLF + DSF experienced slight decrease by $0.3 \%$ for recall (as indicated by the arrow mark in Fig. 5a). 
Table 8 Result of the experiment using electronics dataset

\begin{tabular}{|c|c|c|c|c|c|c|c|c|c|c|}
\hline \multirow{3}{*}{$\begin{array}{l}\text { Feature } \\
\text { selection } \\
\text { method }\end{array}$} & \multirow[t]{3}{*}{ ML algorithm } & \multirow{2}{*}{\multicolumn{3}{|c|}{$\mathrm{BF}$}} & \multicolumn{6}{|c|}{ Proposed feature } \\
\hline & & & & & \multicolumn{3}{|l|}{ SLF } & \multicolumn{3}{|c|}{$S L F+D S F$} \\
\hline & & Prec & $\operatorname{Rec}$ & F-meas & Prec & $\operatorname{Rec}$ & F-meas & Prec & $\operatorname{Rec}$ & F-meas \\
\hline \multirow[t]{7}{*}{ None } & Bayes Net & 0.632 & 0.481 & 0.517 & 0.674 & 0.519 & 0.570 & 0.733 & 0.752 & 0.741 \\
\hline & Naïve Bayes & 0.595 & 0.595 & 0.595 & 0.674 & 0.519 & 0.570 & 0.678 & 0.495 & 0.549 \\
\hline & Logistic & 0.544 & 0.544 & 0.544 & 0.641 & 0.740 & 0.687 & 0.628 & 0.657 & 0.642 \\
\hline & MLP & 0.701 & 0.722 & 0.710 & 0.707 & 0.750 & 0.724 & 0.712 & 0.733 & 0.722 \\
\hline & $J 48$ & 0.607 & 0.658 & 0.629 & 0.651 & 0.798 & 0.717 & 0.743 & 0.733 & 0.738 \\
\hline & Random Forest & 0.660 & 0.747 & 0.670 & ${ }^{*} 0.792$ & ${ }^{*} 0.817$ & ${ }^{*} 0.758$ & 0.823 & 0.646 & 0.752 \\
\hline & Random Tree & 0.689 & 0.684 & 0.686 & 0.730 & 0.750 & 0.739 & 0.757 & 0.762 & ${ }^{*} 0.760$ \\
\hline \multirow[t]{7}{*}{ CA } & Bayes Net & 0.632 & 0.481 & 0.517 & 0.674 & 0.519 & 0.570 & 0.733 & 0.752 & 0.741 \\
\hline & Naïve Bayes & 0.595 & 0.595 & 0.595 & 0.674 & 0.519 & 0.570 & 0.678 & 0.495 & 0.549 \\
\hline & Logistic & 0.544 & 0.544 & 0.544 & 0.641 & 0.740 & 0.687 & 0.628 & 0.657 & 0.642 \\
\hline & MLP & 0.701 & 0.722 & 0.710 & 0.707 & 0.750 & 0.724 & 0.712 & 0.733 & 0.722 \\
\hline & $J 48$ & 0.607 & 0.658 & 0.629 & 0.651 & 0.798 & 0.717 & 0.743 & 0.733 & 0.738 \\
\hline & Random Forest & 0.660 & 0.747 & 0.670 & ${ }^{*} 0.792$ & ${ }^{*} 0.817$ & $*^{*} 0.758$ & 0.646 & 0.752 & 0.695 \\
\hline & Random Tree & 0.689 & 0.684 & 0.686 & 0.730 & 0.750 & 0.739 & 0.757 & 0.762 & ${ }^{*} 0.760$ \\
\hline \multirow[t]{7}{*}{$G R$} & Bayes Net & 0.632 & 0.481 & 0.517 & 0.674 & 0.519 & 0.570 & 0.733 & 0.752 & 0.741 \\
\hline & Naïve Bayes & 0.595 & 0.595 & 0.595 & 0.674 & 0.519 & 0.570 & 0.678 & 0.495 & 0.549 \\
\hline & Logistic & 0.544 & 0.544 & 0.544 & 0.641 & 0.740 & 0.687 & 0.628 & 0.657 & 0.642 \\
\hline & MLP & 0.701 & 0.722 & 0.710 & 0.707 & 0.750 & 0.724 & 0.712 & 0.733 & 0.722 \\
\hline & $J 48$ & 0.607 & 0.658 & 0.629 & 0.651 & 0.798 & 0.717 & 0.743 & 0.733 & 0.738 \\
\hline & Random Forest & 0.660 & 0.747 & 0.670 & 0.792 & 0.817 & 0.758 & 0.646 & 0.752 & 0.695 \\
\hline & Random Tree & 0.689 & 0.684 & 0.686 & 0.730 & 0.750 & 0.739 & 0.757 & 0.762 & ${ }^{*} 0.760$ \\
\hline \multirow[t]{7}{*}{ IG } & Bayes Net & 0.632 & 0.481 & 0.517 & 0.674 & 0.519 & 0.570 & 0.733 & 0.752 & 0.741 \\
\hline & Naïve Bayes & 0.595 & 0.595 & 0.595 & 0.674 & 0.519 & 0.570 & 0.678 & 0.495 & 0.549 \\
\hline & Logistic & 0.544 & 0.544 & 0.544 & 0.641 & 0.740 & 0.687 & 0.628 & 0.657 & 0.642 \\
\hline & MLP & 0.701 & 0.722 & 0.710 & 0.707 & 0.750 & 0.724 & 0.712 & 0.733 & 0.722 \\
\hline & $J 48$ & 0.607 & 0.658 & 0.629 & 0.651 & 0.798 & 0.717 & 0.743 & 0.733 & 0.738 \\
\hline & Random Forest & 0.660 & 0.747 & 0.670 & ${ }^{*} 0.792$ & ${ }^{*} 0.817$ & ${ }^{*} 0.758$ & 0.646 & 0.752 & 0.695 \\
\hline & Random Tree & 0.689 & 0.684 & 0.686 & 0.730 & 0.750 & 0.739 & 0.757 & 0.762 & ${ }^{*} 0.760$ \\
\hline \multirow[t]{7}{*}{ OneR } & Bayes Net & 0.632 & 0.481 & 0.517 & 0.674 & 0.519 & 0.570 & 0.707 & 0.771 & 0.730 \\
\hline & Naïve Bayes & 0.595 & 0.595 & 0.595 & 0.674 & 0.519 & 0.570 & 0.652 & 0.790 & 0.715 \\
\hline & Logistic & 0.544 & 0.544 & 0.544 & 0.641 & 0.740 & 0.687 & 0.654 & ${ }^{*} 0.800$ & 0.720 \\
\hline & MLP & 0.701 & 0.722 & 0.710 & 0.707 & 0.750 & 0.724 & 0.712 & 0.733 & 0.722 \\
\hline & $J 48$ & 0.607 & 0.658 & 0.629 & 0.651 & 0.798 & 0.717 & 0.743 & 0.733 & 0.738 \\
\hline & Random Forest & 0.660 & 0.747 & 0.670 & 0.792 & 0.817 & 0.758 & 0.714 & 0.714 & 0.714 \\
\hline & Random Tree & 0.689 & 0.684 & 0.686 & 0.730 & 0.750 & 0.739 & 0.692 & 0.686 & 0.689 \\
\hline \multirow[t]{7}{*}{ PCA } & Bayes Net & 0.560 & 0.544 & 0.552 & 0.648 & 0.683 & 0.665 & 0.733 & 0.752 & 0.741 \\
\hline & Naïve Bayes & 0.590 & 0.620 & 0.604 & 0.648 & 0.683 & 0.665 & 0.679 & 0.619 & 0.645 \\
\hline & Logistic & 0.550 & 0.633 & 0.589 & 0.648 & 0.779 & 0.707 & 0.644 & 0.743 & 0.690 \\
\hline & MLP & 0.569 & 0.532 & 0.549 & 0.648 & 0.779 & 0.707 & 0.621 & 0.629 & 0.625 \\
\hline & $J 48$ & 0.633 & 0.620 & 0.627 & 0.651 & 0.798 & 0.717 & 0.652 & 0.790 & 0.715 \\
\hline & Random Forest & 0.564 & 0.696 & 0.623 & 0.649 & 0.788 & 0.712 & 0.648 & 0.762 & 0.700 \\
\hline & Random Tree & 0.653 & 0.684 & 0.666 & 0.720 & 0.731 & 0.725 & 0.683 & 0.629 & 0.652 \\
\hline
\end{tabular}


Table 9 Result of the experiment using automobile dataset

\begin{tabular}{|c|c|c|c|c|c|c|c|c|c|c|}
\hline \multirow{3}{*}{$\begin{array}{l}\text { Feature } \\
\text { selection } \\
\text { method }\end{array}$} & \multirow[t]{3}{*}{ ML algorithm } & \multirow{2}{*}{\multicolumn{3}{|c|}{$\mathrm{BF}$}} & \multicolumn{6}{|c|}{ Proposed feature } \\
\hline & & & & & \multicolumn{3}{|l|}{ SLF } & \multicolumn{3}{|c|}{$S L F+D S F$} \\
\hline & & Prec & $\operatorname{Rec}$ & F-meas & Prec & $\operatorname{Rec}$ & F-meas & Prec & $\operatorname{Rec}$ & F-meas \\
\hline \multirow[t]{7}{*}{ None } & Bayes Net & 0.664 & 0.664 & 0.664 & 0.764 & 0.759 & 0.762 & 0.786 & 0.818 & 0.800 \\
\hline & Naïve Bayes & 0.701 & 0.770 & 0.735 & 0.764 & 0.759 & 0.762 & 0.786 & 0.818 & 0.800 \\
\hline & Logistic & 0.700 & 0.752 & 0.724 & 0.751 & 0.796 & 0.772 & 0.779 & 0.847 & 0.801 \\
\hline & MLP & 0.739 & 0.796 & 0.761 & 0.782 & 0.810 & 0.795 & 0.770 & 0.810 & 0.788 \\
\hline & $J 48$ & 0.681 & 0.761 & 0.719 & ${ }^{*} 0.796$ & ${ }^{*} 0.847$ & ${ }^{*} 0.811$ & 0.779 & 0.847 & 0.801 \\
\hline & Random Forest & 0.689 & 0.814 & 0.747 & 0.740 & 0.847 & 0.790 & 0.740 & 0.847 & 0.790 \\
\hline & Random Tree & 0.736 & 0.708 & 0.721 & 0.773 & 0.788 & 0.781 & 0.776 & 0.766 & 0.771 \\
\hline \multirow[t]{7}{*}{ CA } & Bayes Net & 0.707 & 0.770 & 0.735 & 0.764 & 0.759 & 0.762 & 0.786 & 0.818 & 0.800 \\
\hline & Naïve Bayes & 0.664 & 0.664 & 0.664 & 0.764 & 0.759 & 0.762 & 0.786 & 0.818 & 0.800 \\
\hline & Logistic & 0.700 & 0.752 & 0.724 & 0.751 & 0.796 & 0.772 & 0.779 & 0.847 & 0.801 \\
\hline & MLP & 0.739 & 0.796 & 0.761 & 0.782 & 0.810 & 0.795 & 0.770 & 0.810 & 0.788 \\
\hline & $J 48$ & 0.681 & 0.761 & 0.719 & ${ }^{*} 0.796$ & ${ }^{*} 0.847$ & 0.811 & 0.779 & 0.847 & 0.801 \\
\hline & Random Forest & 0.689 & 0.814 & 0.747 & 0.740 & ${ }^{*} 0.847$ & 0.790 & 0.740 & 0.847 & 0.790 \\
\hline & Random Tree & 0.736 & 0.708 & 0.721 & 0.773 & 0.788 & 0.781 & 0.776 & 0.766 & 0.771 \\
\hline \multirow[t]{7}{*}{ GR } & Bayes Net & 0.707 & 0.770 & 0.735 & 0.764 & 0.759 & 0.762 & 0.786 & 0.818 & 0.800 \\
\hline & Naïve Bayes & 0.664 & 0.664 & 0.664 & 0.764 & 0.759 & 0.762 & 0.786 & 0.818 & 0.800 \\
\hline & Logistic & 0.700 & 0.752 & 0.724 & 0.751 & 0.796 & 0.772 & 0.779 & 0.847 & 0.801 \\
\hline & MLP & 0.739 & 0.796 & 0.761 & 0.782 & 0.810 & 0.795 & 0.770 & 0.81 & 0.788 \\
\hline & $J 48$ & 0.681 & 0.761 & 0.719 & ${ }^{*} 0.796$ & ${ }^{*} 0.847$ & ${ }^{*} 0.811$ & 0.779 & 0.847 & 0.801 \\
\hline & Random Forest & 0.689 & 0.814 & 0.747 & 0.740 & ${ }^{*} 0.847$ & 0.790 & 0.740 & 0.847 & 0.790 \\
\hline & Random Tree & 0.736 & 0.708 & 0.721 & 0.773 & 0.788 & 0.781 & 0.776 & 0.766 & 0.771 \\
\hline \multirow[t]{7}{*}{ IG } & Bayes Net & 0.707 & 0.770 & 0.735 & 0.764 & 0.759 & 0.762 & 0.786 & 0.818 & 0.800 \\
\hline & Naïve Bayes & 0.664 & 0.664 & 0.664 & 0.764 & 0.759 & 0.762 & 0.786 & 0.818 & 0.800 \\
\hline & Logistic & 0.700 & 0.752 & 0.724 & 0.751 & 0.796 & 0.772 & 0.779 & 0.847 & 0.801 \\
\hline & MLP & 0.739 & 0.796 & 0.761 & 0.782 & 0.810 & 0.795 & 0.770 & 0.810 & 0.788 \\
\hline & $J 48$ & 0.681 & 0.761 & 0.719 & ${ }^{*} 0.796$ & ${ }^{*} 0.847$ & $*^{*} 0.811$ & 0.779 & 0.847 & 0.801 \\
\hline & Random Forest & 0.689 & 0.814 & 0.747 & 0.740 & ${ }^{*} 0.847$ & 0.790 & 0.740 & 0.847 & 0.790 \\
\hline & Random Tree & 0.736 & 0.708 & 0.721 & 0.773 & 0.788 & 0.781 & 0.776 & 0.766 & 0.771 \\
\hline \multirow[t]{7}{*}{ OneR } & Bayes Net & 0.664 & 0.664 & 0.664 & 0.764 & 0.759 & 0.762 & 0.786 & 0.818 & 0.800 \\
\hline & Naïve Bayes & 0.728 & 0.717 & 0.722 & 0.764 & 0.759 & 0.762 & 0.786 & 0.818 & 0.800 \\
\hline & Logistic & 0.700 & 0.752 & 0.724 & 0.751 & 0.796 & 0.772 & 0.779 & 0.847 & 0.801 \\
\hline & MLP & 0.739 & 0.796 & 0.761 & 0.782 & 0.810 & 0.795 & 0.77 & 0.81 & 0.788 \\
\hline & $J 48$ & 0.681 & 0.761 & 0.719 & *0.796 & ${ }^{*} 0.847$ & $*^{*} 0.811$ & 0.779 & 0.847 & 0.801 \\
\hline & Random Forest & 0.689 & 0.814 & 0.747 & 0.740 & ${ }^{*} 0.847$ & 0.790 & 0.74 & 0.847 & 0.790 \\
\hline & Random Tree & 0.736 & 0.708 & 0.721 & 0.773 & 0.788 & 0.781 & 0.776 & 0.766 & 0.771 \\
\hline \multirow[t]{7}{*}{ PCA } & Bayes Net & 0.681 & 0.761 & 0.719 & 0.770 & 0.810 & 0.788 & 0.806 & *0.854 & 0.816 \\
\hline & Naïve Bayes & 0.749 & 0.743 & 0.746 & 0.770 & 0.810 & 0.788 & 0.806 & $* 0.854$ & 0.816 \\
\hline & Logistic & 0.688 & 0.805 & 0.742 & 0.740 & 0.847 & 0.790 & 0.740 & 0.847 & 0.790 \\
\hline & MLP & 0.700 & 0.752 & 0.724 & 0.742 & 0.759 & 0.750 & 0.782 & 0.832 & 0.802 \\
\hline & $J 48$ & 0.691 & 0.823 & 0.751 & 0.738 & 0.832 & 0.782 & 0.740 & 0.847 & 0.790 \\
\hline & Random Forest & 0.688 & 0.805 & 0.742 & 0.740 & ${ }^{*} 0.847$ & 0.790 & 0.740 & 0.847 & 0.790 \\
\hline & Random Tree & 0.741 & 0.752 & 0.747 & 0.766 & 0.766 & 0.766 & ${ }^{*} 0.825$ & 0.839 & ${ }^{*} 0.831$ \\
\hline
\end{tabular}

\section{Limitation of the study and the future work}

SLF extraction is based on a word sense disambiguation technique that relies on WordNet similarity algorithms. Therefore, the result depends on the effectiveness of 


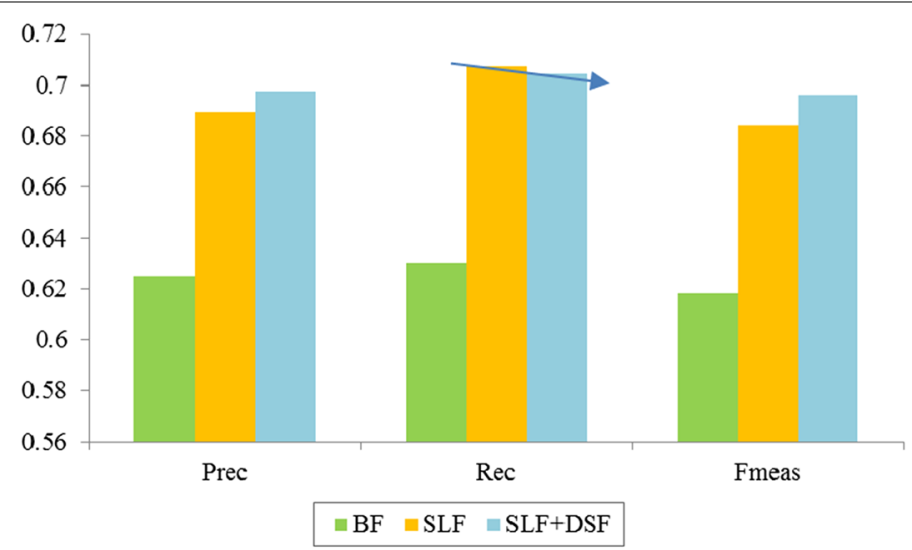

a Electronic Dataset

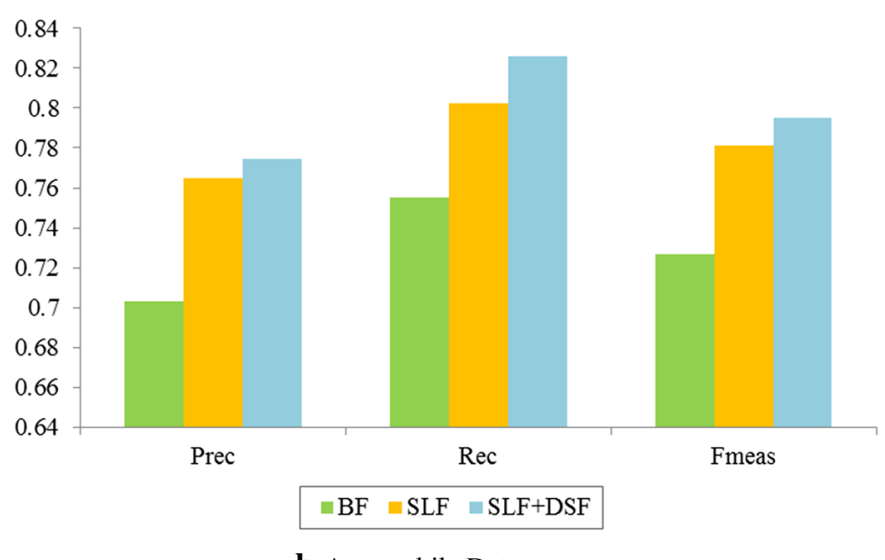

b Automobile Dataset

Fig. 5 Average performance of our proposed features compared with baseline features

Table 10 Technique for determining pivot word

\begin{tabular}{ll}
\hline Study & Rule for determining pivot word \\
\hline Senticircle [37] & Simply pick word that has POS tags NN in tweet \\
This study & $N N+$ similarity algorithm \\
\hline
\end{tabular}

the algorithms. Meanwhile, for SLF + DSF, the implementation is based on a Senticircle technique [37]. In this study, senticircle has an important role to adjust sentiment value of an opinion word based on its product domain. The value of cts $_{i}$ that is the result of SLF has a role in determining sentiment orientation of an opinion word by assigning the value of $\theta_{i}$. More importantly, pivot word $p w_{k}$ is responsible for assigning the rate of the adjustment. Compare to Saif's technique in determining pivot word [37], this study has actually provided extension as seen in Table 10.

The extension and the adopted technique of SLF + DSF yields slight increase in performance metrics compared with SLF. In Electronic dataset, on the contrary, recall 
experienced slight decrease (see Fig. 3a). We hypothesize that pivot word is responsible for this result. Therefore in our future work we will develop technique to determine pivot word. We hypothesize that pivot word is product feature called aspect. We will develop rule to extract product aspect and carry a more fine grain SA task based on pair of aspect and opinion word to provide better increase in performance metrics. In the future work, we also plan to extent the implementation using Python and $\mathrm{R}$ language and big data platforms e.g. Hadoop, Sparkle.

\title{
Conclusion
}

We have implemented the proposed semantic features extraction namely SLF and DSF, which have achieved better performance on supervised SA task. The performance of the proposed features was evaluated using several machine learning algorithms and feature selection methods of WEKA compared with a baseline features. SLF favorably escalate the performance of SA task by $6.2 \%, 6.1 \%$, and $6.0 \%$ for precision, recall, and F-Measure respectively. Meanwhile, SLF+DSF successfully enhance the performance of supervised SA by $7.1 \%, 7.2 \%$, and $7.4 \%$ for precision, recall and F-Measure.

\begin{abstract}
Abbreviations
OPRs: online product reviews; SA: sentiment analysis; ML: machine learning; BOW: bag of words; CBOW: continuous bag of words; WSD: word sense disambiguation; SLF: sentence level features; DSF: domain sensitive features; MPQA: multi perspective question answering; RF: Random Forest; SVM: Support Vector Machine; CNN: Convolutional Neural Network; POS: part of speech; LCS: Least Common Subsumer; MLP: multi layer perceptron; BF: baseline feature; CA: classifier attribute evaluator; GR: gain ratio attribute evaluator; IG: information gain attribute evaluator; OneR: one rule attribute evaluator; PCA: principal component analysis.
\end{abstract}

\section{Acknowledgements}

We would like to thank both Institut Teknologi Sepuluh Nopember and Universitas Muhammadiyah Jember for supporting this work by providing laboratory for running the experiment.

\section{Authors' contributions}

BSR developed the methodology and designed the experiment. BSR also analysed the result and wrote the manuscript under the supervision of RS and CF as academic supervisors. All authors read and approved the final manuscript.

Funding

Not applicable.

Availability of data and materials

The raw dataset used in this study is publicly available and the source is included in the manuscript.

\section{Competing interests}

The authors declare that they have no competing interests.

\section{Author details}

${ }^{1}$ Department of Informatics, Institut Teknologi Sepuluh Nopember, Surabaya, Indonesia. ${ }^{2}$ Department of Electrical

Engineering, Universitas Muhammadiyah Jember, Jember, Indonesia.

Received: 2 June 2019 Accepted: 27 August 2019

Published online: 12 September 2019

\section{References}

1. Sridhar S, Srinivasan R. Social influence effects in online product ratings. J Mark. 2012;76(5):70-88.

2. Zheng L, Wang H, Gao S. Sentimental feature selection for sentiment analysis of Chinese online reviews. Int J Mach Learn Cybern. 2018;9:75-84.

3. Rintyarna BS, Sarno R, Fatichah C. Enhancing the performance of sentiment analysis task on product reviews by handling both local and global context. Int J Inf Decis Sci; 2018 (in press).

4. Budiharto W, Meiliana M. Prediction and analysis of Indonesia presidential election from Twitter using sentiment analysis. J Big Data. 2018;5:1-10.

5. Hsieh WTM. eWOM persuasiveness: do eWOM platforms and product type matter? Electron Commer Res. 2015;57:509-41. 
6. Tsao $\mathrm{H}$, Chen $\mathrm{M}$. The asymmetric effect of review valence on numerical rating: a viewpoint from a sentiment analysis of users of TripAdvisor. 2019;43(2):283-300.

7. Saad S, Saberi B. Sentiment analysis or opinion mining: a review. Int J Adv Sci Eng Inf Technol. 2017;7(5):1660.

8. Baccianella FSS, Esuli A. SentiwordNet 3.0: an enhanced lexical resource for sentiment analysis and opinion mining. In: Proceedings of the 9th conference on language resources and evaluation; 2010. p. 2200-4.

9. Taboada M, Brooke J, Tofiloski M. Lexicon-based methods for sentiment analysis. Comput Linguist. 2011;37(September 2010):267-307.

10. Wilson PHT, Wiebe J. Recognizing contextual polarity in phrase-level sentiment analysis. In: Proceedings of human language technology conference and conference on empirical methods in natural language processing. Vancouver, Br. Columbia, Canada; 2005.

11. Qiu G, Liu B, Bu J, Chen C. Opinion word expansion and target extraction through double propagation. Comput Linguist. 2011;37:9-27.

12. Cambria E, Havasi C, Hussain A. SenticNet 2: a semantic and affective resource for opinion mining and sentiment analysis. In: Twenty-fifth international FLAIRS conference; 2012. p. 202-7.

13. Mohammad SM, Turney PD. NRC emotion lexicon. Ottawa: National Research Council; 2013. p. 1-234.

14. Medhat W, Hassan A, Korashy H. Sentiment analysis algorithms and applications: a survey. Ain Shams Eng J. 2014:5(4):1093-113.

15. Staš J, Juhár J, Hládek D. Classification of heterogeneous text data for robust domain-specific language modeling. EURASIP J Audio Speech Music Process. 2014. https://doi.org/10.1186/1687-4722-2014-14.

16. Al Amrani Y, Lazaar M, El Kadiri KE. Random forest and support vector machine based hybrid approach to sentiment analysis. Procedia Comput Sci. 2018;127:511-20.

17. Yin Y, Jin Z. Document sentiment classification based on the word embedding. In: 4th international conference on mechatronics, materials, chemistry and computer engineering; 2015. p. 456-61.

18. Gezici G, Dehkharghani R, Yanikoglu B, Tapucu D, Saygin Y. SU-Sentilab : a classification system for sentiment analysis in Twitter. In: Seventh international workshop on semantic evaluation, vol. 2, no. SemEval; 2013. p. 471-7.

19. Yoo SY, Song Jl, Jeong OR. Social media contents based sentiment analysis and prediction system. Expert Syst Appl. 2018;105:102-11.

20. Sinha R, Mihalcea R. Unsupervised graph-based word sense disambiguation using measures of word semantic similarity. In: International conference on semantic computing (ICSC 2007); 2007. p. 363-9.

21. Pandey H, Mishra AK, Kumar N. Various aspects of sentiment analysis. In: International conference on advanced computing and software engineering; 2019 .

22. Vyas $V$, Uma V. Approaches to sentiment analysis on product reviews. In: Sentiment analysis and knowledge discovery in contemporary business, IGI Global; 2019. p. 15-30.

23. Tang F, Fu L, Yao B, Xu W. Aspect based fine-grained sentiment analysis for online reviews. Inf Sci. 2019;488:190-204.

24. Jo Y, Oh A. Aspect and sentiment unification model for online review analysis. In: Proceedings of the fourth ACM international conference on Web search and data mining; 2011. p. 815-24.

25. García-Pablos A, Cuadros M, Rigau G. W2VLDA: almost unsupervised system for aspect based sentiment analysis. Expert Syst Appl. 2018;91:127-37.

26. Ganu G, Elhadad N, Marian A. Beyond the stars : improving rating predictions using review text content. In: Proceeding of WebDB, no. 9; 2009. p. 1-6.

27. Pontiki M, et al. "SemEval-2016 task 5 : aspect based sentiment analysis. In: Proceedings of the tenth international workshop on semantic evaluation (Se-meval-2016); 2016. p. 19-30.

28. Amplayo RK, Lee S, Song M. Incorporating product description to sentiment topic models for improved aspectbased sentiment analysis. Inf Sci. 2018:454:200-15.

29. Rana TA, Cheah Y. A two-fold rule-based model for aspect extraction. Expert Syst Appl. 2017;89:273-85.

30. Rana TA, Cheah YN. Exploiting sequential patterns to detect objective aspects from online reviews. In: International conference on advanced informatics: concepts, theory and application; 2016.

31. Rintyarna BS, Sarno R, Yuananda AL. Automatic ranking system of university based on technology readiness level using LDA-Adaboost.MH. In: 2018 international conference on information and communications technology (ICOIACT), vol. 2018; 2018. p. 495-9.

32. Miller GA. WordNet: a lexical database for english. Commun ACM. 1995;38(11):39-41.

33. Aliyanto D, Sarno R, Rintyarna BS. Supervised probabilistic latent semantic analysis (SPLSA) for estimating technology readiness level. In: International conference on information \& communication technology and system; 2017. p. 79-84.

34. Banerjee S, Pedersen T. An adapted lesk algorithm for word sense disambiguation using WordNet. Comput Linguist Intell Text Process. 2002;2276:136-45.

35. Saif $\mathrm{H}, \mathrm{He} Y$, Fernandez M, Alani H. Contextual semantics for sentiment analysis of Twitter. Inf Process Manag. 2016;52(1):5-19.

36. Wu Z, Palmer M. Verb semantics and lexical Zhibiao W u. In: Proceedings of the 32nd annual meeting of the association for computational linguistics; 1994. p. 133-8.

37. Saif $\mathrm{H}, \mathrm{He} \mathrm{Y}$, Fernandez M, Alani H. Contextual semantics for sentiment analysis of Twitter. Inf Process Manag. 2014;52(1):5-19.

38. Hall $M$, et al. The WEKA data mining software: an update, vol. 11, no. 1, p. 10-8.

39. McAuley J, Pandey R, Leskovec J. Inferring networks of substitutable and complementary products. In: Proceedings of the 21th ACM SIGKDD international conference on knowledge discovery and data mining. 2015; p. 785-94.

\section{Publisher's Note}

Springer Nature remains neutral with regard to jurisdictional claims in published maps and institutional affiliations. 